\title{
A Mobilidade Dentária e o Impacto na Qualidade de Vida: Um Estudo Piloto
}

\author{
Dental Mobility and the Impact on Quality of Life: A Pilot Study
}

\author{
Arthur Antunes Costa Bezerra \\ Plínio Barbalho Vieira Tavares ${ }^{1}$ \\ Ivone Lima Santana ${ }^{2}$
}

Frederico Silva de Freitas Fernandes ${ }^{2}$

Adriana de Fátima Vasconcelos Pereira ${ }^{3}$

\section{RESUMO}

Objetivo: Avaliar o impacto da mobilidade dentária na qualidade de vida de indivíduos adultos. Metodologia: Trata-se de um estudo transversal no qual foram examinados pacientes, de ambos os sexos, nas clínicas do Curso de Odontologia da UFMA. Avaliou-se um total de 487 dentes, dos quais 138 apresentavam mobilidade. Foram coletados dados pessoais e parâmetros clínicos para obtenção do diagnóstico periodontal. O questionário OHIP-14 (Oral health impact profile) foi utilizado para avaliar o impacto da mobilidade dentária (variando de grau 1 a 3) na qualidade de vida. Realizou-se uma análise descritiva, por meio de frequências absolutas e relativas, dos dados pessoais, dos diagnósticos periodontais e dos valores do OHIP-14. A associação entre as variáveis foi verificada pelo teste Qui-quadrado de Pearson com nível de significância de $5 \%$. Resultados: Observou-se que o impacto na qualidade de vida dos participantes variou entre fraco e médio e que os dentes mais afetados foram os incisivos inferiores e os menos afetados foram os caninos superiores. $O$ grau 1 de mobilidade foi o mais encontrado. Conclusão: De acordo com os dados da pesquisa, foi observado que a mobilidade dentária apresentou um impacto fraco na qualidade de vida dos pacientes.

\section{DESCRITORES}

Mobilidade Dentária. Qualidade de Vida. Inquéritos e Questionários.

\begin{abstract}
Objective: To evaluate the impact of dental mobility on quality of life. Methodology: This cross-sectional study was performed with adult patients of both sexes that were examined at UFMA dental clinics. 487 teeth were evaluated, of which 138 had mobility. personal data and clinical parameters for periodontal diagnosis were collected. The OHIP-14 (Oral Health Impact Profile) questionnaire was used to assess tooth mobility (degree 1 to 3 ) on the quality of life. A descriptive analysis was performed, using absolute and relative frequencies of the personal data, periodontal diagnosis, and OHIP-14 applications. The association between the variables was verified by Pearson's Chi-squared test with significance level of $5 \%$. Results: It was observed that the degree of impact on the quality of life ranged from weak to medium, and that the most affected teeth were the lower incisors and the less affected were the upper canines. Grade 1 of mobility was the most common. Conclusion: According to research findings dental mobility has shown a weak impact on the patients' quality of life.
\end{abstract}

\section{DESCRIPTORS}

Dental Mobility. Quality of Life. Surveys and Questionnaires.

${ }^{1}$ Graduado em Odontologia pela Universidade Federal do Maranhão - UFMA.

${ }^{2}$ Professor(a) Associado(a) do Departamento de Odontologia I, do Curso de Odontologia da Universidade Federal do Maranhão - UFMA.

${ }^{3}$ Professora Associada ao Departamento de Odontologia II, do Curso de Odontologia da Universidade Federal do Maranhão - UFMA. 
conceito de qualidade de vida referese a um movimento dentro das ciências humanas e biológicas no sentido de valorizar parâmetros mais amplos que o controle de sintomas, a diminuição da mortalidade ou o aumento da expectativa de vida ${ }^{1}$. Trata-se de uma definição muito complexa, resultado de um consenso internacional, representando uma visão transcultural, bem como multidimensional, que contempla a influência da saúde física e psicológica, o nível de independência, as relações sociais, as crenças pessoais e a avaliação subjetiva, segundo a Organização Mundial de Saúde².

O termo saúde bucal relacionado à qualidade de vida é comumente utilizado para descrever o impacto que os problemas bucais podem interferir na vida das pessoas ${ }^{3}$. Dentre esses problemas, destacam-se a cárie dentária ${ }^{4,5}$, o traumatismo dental ${ }^{6}$ e a mobilidade dentária ${ }^{7}$.

A mobilidade dentária é caracterizada pelo aumento da amplitude de deslocamento da coroa de um dente, com ou sem a presença de oclusão traumática ${ }^{8}$. Contudo, a mobilidade é um achado muito comum quando o dente está sob trauma oclusal ${ }^{7}$. Migração dentária, dor ou desconforto à mastigação, além de reabsorção de osso alveolar estão associados à mobilidade, quando associada aos hábitos nocivos, como fumar ${ }^{9}$. A mudança de posição dentária e a sensibilidade dolorosa devido à mobilidade são geralmente autoperceptíveis e interferem na qualidade de vida dos indivíduos ${ }^{6}$.

A origem da mobilidade dentária é multifatorial. Quando relacionada ao agravo da doença periodontal apresenta perda óssea e de inserção clínica, além de poder ser influenciada por diferentes condições, tais como: a ausência dentária acompanhada ou não do uso de próteses dentárias; trauma da oclusão primária, secundária e ortodôntica; forças traumáticas em função da perda dentária e migração dos dentes pré-molares e molares, acompanhada pela separação gradual dos dentes superiores anteriores; restaurações e coroas protéticas insatisfatórias; hábitos parafuncionais, como apertamento dental, cêntrico ou excêntrico ${ }^{7,10}$.

Apesar de a mobilidade dentária apresentar etiologia multifatorial e promover uma influência na qualidade de vida ${ }^{11-15}$, poucos estudos abordam diretamente a sua correlação com os dados subjetivos por meio da autopercepção das pessoas. Dentro desse contexto, o objetivo desta pesquisa foi avaliar o impacto da mobilidade dentária na qualidade de vida.

\section{METODOLOGIA}

O estudo transversal foi aprovado pelo Comitê de Ética em Pesquisa da UFMA (Parecer $n^{\circ}$. 275.507). Os voluntários foram devidamente esclarecidos sobre os aspectos envolvidos na realização da pesquisa e assinaram um Termo de consentimento livre e esclarecido. Foram excluídos aqueles que se recusaram à avaliação clínica e em responder ao questionário de qualidade de vida.

Avaliou-se um total de 25 indivíduos adultos, de ambos os sexos e na faixa etária de 28 a 72 anos nas clínicas do Curso de Odontologia da UFMA, considerando as condições sistêmicas e hábitos comportamentais. 
Além dos dados pessoais, foram coletadas as informações sobre os parâmetros clínicos como o índice de placa, Índice de sangramento gengival, Profundidade de sondagem, Nível gengival e Nível de inserção clínica para estabelecer o diagnóstico periodontal ${ }^{16-19}$. A mobilidade dentária foi estabelecida quanto ao grau em: Grau 1 (mobilidade da coroa do dente de 0,2 - 1,0 mm no sentido horizontal), Grau 2 (mobilidade da coroa do dente excedendo $1,0 \mathrm{~mm}$ no sentido horizontal) e Grau 3 (mobilidade horizontal e vertical), utilizando-se 2 cabos de instrumentos ${ }^{20}$.

Foi aplicada a versão brasileira do índice OHIP-14 (Oral Health Impact Profile) para avaliar a qualidade de vida de cada paciente em relação à sua respectiva saúde bucal por meio de questões agrupadas em sete subescalas: limitação funcional, dor física, desconforto psicológico, incapacidade física, psicológica e social e deficiência na realização das atividades cotidianas ${ }^{21}$.

Para calcular o valor individual do OHIP-14 foi utilizado o método ponderado padronizado, que leva em consideração o peso de cada questão. Em seguida, multiplicou-se pela codificação da pergunta conforme a escala do tipo likert (nunca $=0$; raramente $=1$; às vezes $=2$; frequentemente $=3$; sempre $=4$; não sabe = exclusão de todo o formulário). 0 peso de cada item foi gerado pelo método de comparação pareada de Thurstone ${ }^{21}$. 0 somatório de todas as perguntas, para cada indivíduo, compreendeu de 0 a 28 pontos. 0 impacto individual foi classificado em fraco $(0$ a 9), médio (10 a 18) e forte (19 a 28) 22 .

Os dados coletados foram reportados e tabulados usando os programas Microsoft
Office Word 2016 e Microsoft Excel 2016. Utilizou-se o programa Epi Info (Versão 7.2) para calcular as frequências absolutas e relativas dos dados pessoais, diagnósticos periodontais e os valores do OHIP-14. A associação entre as variáveis foi verificada por meio do teste não paramétrico Qui-quadrado de Pearson, com nível de significância de 5\%.

\section{RESULTADOS}

A Tabela 1 mostra as frequências das variáveis da amostra do estudo, enquanto a Tabela 2 demonstra as frequências dos dados clínicos periodontais, além da distribuição dos pacientes de acordo com a quantidade de dentes com mobilidade. $\mathrm{O}$ diagnóstico periodontal mais encontrado foi o de periodontite (19 pessoas). A maioria dos indivídos (11 pessoas) apresentava 5 dentes ou mais com mobilidade.

De 487 dentes avaliados, um total de 138 dentes apresentou algum grau de mobilidade. Na Tabela 3, observou-se que o grau 1 foi predominante em 77 dentes e que os dentes mais afetados foram os incisivos (50 dentes).

O impacto da mobilidade dentária sobre a qualidade de vida dos pacientes variou de fraco (13 pessoas) a médio ( 9 pessoas) pelo OHIP-14. Estes achados foram correlacionados com algumas variáveis da caracterização da amostra (sexo, idade, diagnóstico periodontal, diabetes mellitus, hipertensão e número de dentes com mobilidade), não existindo diferença significativa entre homens e mulheres, em relação ao impacto na qualidade de vida e 
que, em geral, o maior número de indivíduos dentro de cada variável sofreu impacto fraco (Tabela 4).

Quanto às questões do OHIP-14, observou-se que a pergunta 5 (pertencente à subescala de desconforto psicológico) foi a que mais recebeu como resposta a alternativa "sempre" (Tabela 5).

\section{Tabela 1. Caracterização dos participantes do estudo}

\begin{tabular}{|c|c|c|}
\hline \multicolumn{2}{|c|}{ Variável } & \multirow{2}{*}{$\begin{array}{c}\text { Frequência } \\
\mathrm{n} / \% \\
10 / 45,45\end{array}$} \\
\hline \multirow[t]{2}{*}{ Sexo } & Masculino & \\
\hline & Feminino & $12 / 54,55$ \\
\hline \multirow[t]{2}{*}{ Idade } & $15-59$ anos & $17 / 77,27$ \\
\hline & 60 anos ou mais & $05 / 22,73$ \\
\hline \multirow[t]{2}{*}{ Diabetes Mellitus } & Sim & $04 / 18,18$ \\
\hline & não & $18 / 81,82$ \\
\hline \multirow[t]{2}{*}{ Hipertensão Arterial } & Sim & $06 / 27,27$ \\
\hline & Não & $16 / 72,73$ \\
\hline \multirow[t]{2}{*}{ Fumante } & $\operatorname{sim}$ & - \\
\hline & não & $22 / 100$ \\
\hline \multirow[t]{2}{*}{ Ex Fumante } & Sim & $09 / 40,91$ \\
\hline & não & $13 / 59,09$ \\
\hline
\end{tabular}

Tabela 2. Avaliação clínica da condição periodontal

\begin{tabular}{|c|c|c|}
\hline \multicolumn{2}{|l|}{ Variável } & Frequêcia \\
\hline \multirow{2}{*}{$\begin{array}{l}\text { Indice de Placa (O'Leary, Drake, } \\
\text { Naylor, 1972) }\end{array}$} & Até $25 \%$ & - \\
\hline & $26 \%$ ou mais & $22 / 100$ \\
\hline \multirow{2}{*}{$\begin{array}{l}\text { Indice de Sangramento Gengival } \\
\text { (Ainamo; Bay,1975) }\end{array}$} & Até $10 \%$ & $02 / 9,09$ \\
\hline & $11 \%$ ou mais & $20 / 90,91$ \\
\hline \multirow{3}{*}{ Diagnóstico Periodontal* } & Saúde Periodontal & - \\
\hline & $\begin{array}{c}\text { Gengivite Associada ao } \\
\text { Biofilme }\end{array}$ & $03 / 13,64$ \\
\hline & Periodontite & $19 / 86,36$ \\
\hline \multirow{3}{*}{$\begin{array}{l}\text { Número de dentes com } \\
\text { mobilidade }\end{array}$} & Até 2 & $07 / 31,82$ \\
\hline & 3 a 4 & $04 / 18,18$ \\
\hline & 5 ou mais & $11 / 50,00$ \\
\hline
\end{tabular}

* Classificação das Doenças Periodontais Academia Americana de Periodontologia $(2018)^{19}$. 
Tabela 3. Análise descritiva das variáveis examinadas nos dentes com mobilidade

\begin{tabular}{|c|c|c|c|}
\hline \multirow{3}{*}{$\begin{array}{c}\text { Classe de } \\
\text { dentes }\end{array}$} & \multirow{3}{*}{$\begin{array}{c}\text { Grau de } \\
\text { mobilidade }\end{array}$} & \multicolumn{2}{|c|}{ Sexo } \\
\hline & & Masculino & Feminino \\
\hline & & \multicolumn{2}{|c|}{$n / \%$} \\
\hline \multirow{3}{*}{ Incisivos } & Grau 1 & 14 / 18,19 & $15 / 19,49$ \\
\hline & Grau 2 & $05 / 11,11$ & $11 / 24,44$ \\
\hline & Grau 3 & $03 / 18,75$ & $02 / 12,5$ \\
\hline \multirow{3}{*}{ Caninos } & Grau 1 & $03 / 3,89$ & $04 / 5,19$ \\
\hline & Grau 2 & $02 / 4,45$ & $03 / 6,67$ \\
\hline & Grau 3 & $01 / 6,25$ & $01 / 6,25$ \\
\hline \multirow{3}{*}{ Pré-molares } & Grau 1 & $12 / 15,58$ & $10 / 12,99$ \\
\hline & Grau 2 & $04 / 8,88$ & $07 / 15,55$ \\
\hline & Grau 3 & - & $04 / 25$ \\
\hline \multirow{3}{*}{ Molares } & Grau 1 & $10 / 12,99$ & $09 / 11,68$ \\
\hline & Grau 2 & $01 / 2,23$ & $12 / 26,67$ \\
\hline & Grau 3 & $02 / 12,5$ & $03 / 18,75$ \\
\hline
\end{tabular}

Tabela 4. Associação do OHIP-14 com as variáveis de caracterização da amostra

\begin{tabular}{|c|c|c|c|c|c|}
\hline \multirow{3}{*}{ Variável } & \multicolumn{4}{|c|}{ Impacto na qualidade de vida } & \multirow[b]{3}{*}{ p-valor* } \\
\hline & \multicolumn{2}{|c|}{ Fraco } & \multicolumn{2}{|c|}{ Médio } & \\
\hline & $\mathrm{n}$ & $\%$ & $\mathrm{n}$ & $\%$ & \\
\hline \multicolumn{6}{|l|}{ Sexo } \\
\hline Homens & 06 & 46,15 & 04 & 44,44 & \multirow[t]{2}{*}{0,639} \\
\hline Mulheres & 07 & 53,85 & 05 & 55,56 & \\
\hline \multicolumn{6}{|l|}{ Idade } \\
\hline 15 a 59 anos & 09 & 69,23 & 08 & 88,89 & \multirow[t]{2}{*}{0,293} \\
\hline 60 anos ou mais & 04 & 30,77 & 01 & 11,11 & \\
\hline \multicolumn{6}{|c|}{ Diagnóstico Periodontal } \\
\hline Gengivite & 02 & 15,38 & 01 & 11,11 & \multirow[t]{2}{*}{0,641} \\
\hline Periodontite & 11 & 84,62 & 08 & 89,89 & \\
\hline \multicolumn{6}{|l|}{ Diabetes Mellitus } \\
\hline Sim & 04 & 30,77 & - & - & \multirow[t]{2}{*}{0,097} \\
\hline Não & 09 & 69,23 & 09 & 100,00 & \\
\hline \multicolumn{6}{|l|}{ Hipertensão } \\
\hline Sim & 05 & 38,46 & 01 & 11,11 & \multirow[t]{2}{*}{0,178} \\
\hline não & 08 & 61,54 & 08 & 88,89 & \\
\hline \multicolumn{6}{|l|}{ Número de dentes } \\
\hline Até 2 & 04 & 30,77 & 03 & 33,33 & \multirow[t]{3}{*}{0,2418} \\
\hline 3 a 4 & 02 & 15,38 & 02 & 22,22 & \\
\hline 5 ou mais & 07 & 53,85 & 04 & 44,44 & \\
\hline
\end{tabular}

ॠTeste do Qui-quadrado de Pearson. 
Tabela 5. Distribuição de frequência das respostas dos participantes para cada questão do OHIP-14

\begin{tabular}{|c|c|c|c|c|c|}
\hline & $\begin{array}{c}\text { Nunca } \\
\mathrm{n} / \%\end{array}$ & $\begin{array}{c}\text { Raramente } \\
\mathrm{n} / \%\end{array}$ & $\begin{array}{c}\text { Às vezes } \\
\mathrm{n} / \%\end{array}$ & $\begin{array}{c}\text { Repetidamente } \\
\mathrm{n} / \%\end{array}$ & $\begin{array}{c}\text { Sempre } \\
\mathrm{n} / \%\end{array}$ \\
\hline $\begin{array}{l}\text { 1-Você teve } \\
\text { problemas para } \\
\text { falar alguma } \\
\text { palavra? }\end{array}$ & $17 / 77,27$ & 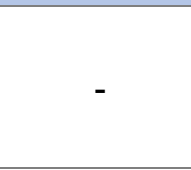 & $04 / 18,18$ & 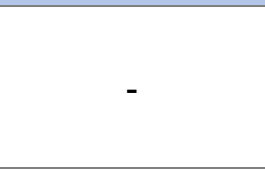 & $01 / 4,55$ \\
\hline $\begin{array}{l}\text { 2-Você sentiu que } \\
\text { o sabor dos } \\
\text { alimentos tem } \\
\text { piorado? }\end{array}$ & $12 / 54,55$ & $03 / 13,64$ & $05 / 22,73$ & $01 / 4,55$ & $01 / 4,55$ \\
\hline $\begin{array}{l}\text { 3-Você sentiu } \\
\text { dores em sua boca } \\
\text { ou nos seus } \\
\text { dentes? }\end{array}$ & $04 / 18,18$ & $04 / 18,18$ & $11 / 50,00$ & $02 / 9,09$ & $01 / 4,55$ \\
\hline $\begin{array}{l}\text { 4-Você se sentiu } \\
\text { incomodado(a) ao } \\
\text { comer algum } \\
\text { alimento? }\end{array}$ & $6 / 27,27$ & $03 / 13,64$ & $08 / 36,36$ & $01 / 4,55$ & $04 / 18,18$ \\
\hline $\begin{array}{l}\text { 5-Você ficou } \\
\text { preocupado(a)? }\end{array}$ & $01 / 4,55$ & $01 / 4,55$ & $05 / 22,73$ & $02 / 9,09$ & $13 / 59,09$ \\
\hline $\begin{array}{l}\text { 6-Você se sentiu } \\
\text { estressado(a)? }\end{array}$ & $11 / 50,00$ & $01 / 4,55$ & $07 / 31,82$ & - & $03 / 13,64$ \\
\hline $\begin{array}{l}\text { 7-Sua alimentação } \\
\text { ficou prejudicada? }\end{array}$ & $07 / 31,82$ & $02 / 9,09$ & $09 / 40,91$ & $01 / 4,55$ & $03 / 13,64$ \\
\hline $\begin{array}{l}\text { 8-Você teve que } \\
\text { parar suas } \\
\text { refeições? }\end{array}$ & $19 / 86,36$ & - & $02 / 9,09$ & - & $01 / 4,55$ \\
\hline $\begin{array}{l}\text { 9-Você encontrou } \\
\text { dificuldade para } \\
\text { relaxar? }\end{array}$ & $11 / 50,00$ & $03 / 13,64$ & $07 / 31,82$ & - & $01 / 4,55$ \\
\hline $\begin{array}{l}\text { 10-Você se sentiu } \\
\text { envergonhado(a)? }\end{array}$ & $08 / 36,36$ & $02 / 9,09$ & $04 / 18,18$ & - & $08 / 36,36$ \\
\hline $\begin{array}{l}11 \text {-Você ficou } \\
\text { irritado(a) com } \\
\text { outras pessoas? }\end{array}$ & $12 / 54,55$ & $01 / 4,55$ & $07 / 31,82$ & $01 / 4,55$ & $01 / 4,55$ \\
\hline $\begin{array}{l}12-\text { Você teve } \\
\text { dificuldade para } \\
\text { realizar suas } \\
\text { atividades diárias? }\end{array}$ & $15 / 68,18$ & $02 / 9,09$ & $05 / 22,73$ & - & - \\
\hline $\begin{array}{l}\text { 13-Você sentiu que } \\
\text { a vida, em geral, } \\
\text { ficou pior? }\end{array}$ & $13 / 59,09$ & $03 / 13,64$ & $4 / 18,18$ & $01 / 4,55$ & $01 / 4,55$ \\
\hline $\begin{array}{l}14-\text { Você ficou } \\
\text { totalmente incapaz } \\
\text { de fazer suas } \\
\text { atividades diárias? }\end{array}$ & $18 / 81,82$ & $02 / 9,09$ & $02 / 9,09$ & - & - \\
\hline
\end{tabular}




\section{DISCUSSÃO}

Uma grande importância tem sido dada à percepção do indivíduo sobre a sua condição bucal, pois a avaliação subjetiva proporciona informações que complementam o exame clínico realizado pelo profissional ${ }^{23}$.

Considerando a condição bucal, a mudança de posição dentária e a sensibilidade dolorosa ocasionadas pela mobilidade, são geralmente autoperceptíveis e podem influenciar no dia a dia dos indivíduos ${ }^{6}$. Dessa forma, este estudo buscou avaliar o impacto da mobilidade dentária na qualidade de vida.

O impacto da mobilidade dentária na qualidade de vida, pelo OHIP-14 dos participantes, variou de fraco a médio. Isso se assemelha ao que foi encontrado em outro estudo $^{24}$, no qual relacionaram as condições bucais de 218 hipertensos e diabéticos com suas respectivas qualidades de vida obtendo, em sua maioria, impacto fraco. Outros pesquisadores também encontraram resultados semelhantes em seu estudo ${ }^{25}$, ao determinar o impacto da saúde bucal e das variáveis socioeconômicas sobre a qualidade de vida em uma amostra de 18 brasileiros de baixa renda.

Quando as questões do OHIP-14 foram analisadas isoladamente, percebeuse que a alternativa "sempre" foi a mais respondida para a pergunta 05 (Tabela 5). Dados similares foram observados em um estudo realizado por Oliveira et al. ${ }^{24}$, no qual avaliaram a percepção dos pacientes quanto à qualidade de vida, condição periodontal, perda dentária e uso ou não de prótese dentária.

No presente estudo, as dimensões mais pontuadas do OHIP-14 foram "desconforto psicológico" e "dor física". Ao comparar com os resultados de Melo $^{6}$, por exemplo, percebeuse que existiu uma pequena diferença em que o "desconforto psicológico" e a "limitação funcional" foram as dimensões que registraram as maiores pontuações.

Um achado importante desta pesquisa foi que 11 pacientes, em sua maioria homens, apresentavam 5 dentes ou mais com mobilidade. Entretanto, isso não repercutiu de forma tão significante na qualidade de vida dos mesmos, visto que 7 apresentaram impacto fraco, enquanto apenas 4 sofreram impacto médio.

Quando relacionados os sexos dos indivíduos com os efeitos da mobilidade na qualidade de vida, não foi encontrada diferença significativa entre homens e mulheres, corroborando com os resultados de um estudo realizado em $2013^{6}$, onde se avaliou os impactos das condições bucais autopercebidas com a qualidade de vida de 360 servidores da região metropolitana de Pernambuco, Brasil.

A classe de dente que mais apresentou mobilidade foi a dos incisivos, sendo que 34 eram inferiores e 16 eram superiores. Uma hipótese para justificar este achado foi que os dentes anteriores inferiores estão mais vulneráveis aos danos das doenças periodontais por apresentarem as lâminas alveolares, vestibular e lingual, mais finas e as cristas dos septos interdentários apresentarem espessura, largura e angulação menores ${ }^{8}$.

A face lingual dos incisivos inferiores apresenta maior probabilidade de formar cálculo dental ${ }^{8}$, embora, desempenhe um papel secundário na etiologia das doenças periodontais, fornece uma superfície ideal 
para maior acúmulo de biofilme bacteriano ${ }^{26}$, além de dificultar as práticas adequadas de higiene bucal ${ }^{18}$. Esses resultados e essa hipótese corroboram os dados da Pesquisa Nacional de Saúde Bucal ${ }^{27}$, que constatou que o achado clínico mais relevante encontrado na população adulta brasileira foi o cálculo dental na região dos dentes anteriores inferiores. Deve ser enfatizado que a concentração de fosfolipídeos na saliva dos indivíduos, que são grandes formadores de cálculo dentário, é significativamente maior do que nos indivíduos que formam pouco cálculo, sugerindo a sua importância na formação/calcificação dessa estrutura ${ }^{28}$.

Os caninos foram os dentes que menos apresentaram mobilidade. Isso pode ser explicado pelo fato de que eles são os dentes mais estáveis na dentição. Uma razão para isso é a grande extensão de suas raízes, que recebem uma maior quantidade de tecido periodontal de suporte. Além disso, existe uma redução significativa do risco de perda desses dentes em decorrência de doença periodontal ou lesão traumática, devido à existência de concavidades proximais em suas raízes que ajudam a obter maior ancoragem periodontal. Isso faz com que, geralmente, eles sejam os

\section{REFERÊNCIAS}

1. Fleck MPA, Leal OF, Louzada S, Xavier M, Chachamovich E, Vieira G, et al. Desenvolvimento da versão em português do instrumento de avaliação de qualidade de vida da OMS (WHOQOL-100). Rev Bras Psiquiatr .1999; 21(1):19-28.

2. WHOQOL Group. The World Health Organization Quality of Life Assessment (WHOQOL): Development and general psychometric properties. Social Science e Medicine. 1998;46(12):1569-1585. últimos dentes a serem perdidos em uma dentição doente ${ }^{29}$.

Os pacientes avaliados, em sua maioria, apresentaram diagnóstico de periodontite e dentes com mobilidade grau 3 (Tabela 4). Contudo, ao avaliar isoladamente por classe de dentes o grau 1 foi o mais observado. Resultados semelhantes foram obtidos por outros pesquisadores em um estudo ${ }^{10} \mathrm{com}$ 155 indivíduos que foram diagnosticados com periodontite que apresentaram maior prevalência de dentes com mobilidade grau 3 .

\section{CONCLUSÃO}

De acordo com os dados obtidos, pode-se concluir que existiu a constatação do impacto fraco da mobilidade dentária na qualidade de vida, embora, a questão mais pontuada do OHIP-14 tenha sido a de número 5. Isso demostrou que a mobilidade dental provoca, de certa forma, preocupação aos indivíduos, mas não de forma tão significativa. Portanto, existe a necessidade de se realizar novas pesquisas, atendendo um maior número de pessoas para corroborar os dados preliminares.

3. Locker D, Allen F. What do measures of oral health-related quality of life measure. Community Dent Oral Epidemiol. 2007; 35(6):401-411.

4. Bendo CB, Paiva SM, Varni JW, Vale MP. Oral health-related quality of life and traumatic dental injuries in Brazilian adolescents. Community Dent Oral Epidemiol. 2014; 42(3):216-223. 
5. Abanto J, Carvalho TS, Mendes FM, Wanderley MT, Bönecker M, Raggio DP. Impact of oral diseases and desordens on oral health-related quality of life of preschool children. Community Dent Oral Epidemiol. 2001; 39:105114.

6. Melo ACS. Análise do Impacto das Condições Bucais Autopercebidas na Qualidade de Vida dos Servidores da Região Metropolitana do Tribunal Regional do Trabalho de Pernambuco. [Dissertação de Mestrado]. Instituto Aggeu Magalhães, Fundação Oswaldo Cruz, Recife, 2013. 141p.

7. Fan J, Caton JG. Occlusal trauma and excessive occlusal forces: Narrative review, case definitions, and diagnostic considerations. J Clin Periodontol. 2018;45(Suppl 20):S199-S206.

8. Newman MG, Takei HH., Klokkevold PR, Carranza FA. Periodontia clínica. $9^{a}$ ed., Rio de Janeiro: Guanabara Koogan, 2016.

9. Nogueira-Filho G, Fróes Neto EB, Casati MZ, Reis SR, Tunes RS, Tunes UR. et al. Nicotine effects on alveolar bone changes induced by occlusal trauma: A histometric study in rats. J Periodontol. 2004; 75:348-352.

10. Soares RSC, Gusmão ES, Cimões R, Silva LS, Moreira RAL, Silva VLC. et al. Movilidad dentaria en la periodontitis crónica. Acta Odontológica Venezoelana. 2011; 49(4):2122.

11. Needleman I, McGrath C, Floyd P, Biddle A. Impact of oral health on the life quality of periodontal patients. J Clin Periodontol. 2004; 31(6):454-457.

12. Valente MIB. As consequências da doença periodontal na qualidade de vida relacionada à saúde bucal e implicações para a definição de necessidade de tratamento. [Tese de Doutorado]: Rio de Janeiro. 2003. 141 p.

13. Acharya S, Bhat PV, Acharya S. Factors affecting oral health-related quality of life among pregnant women. Int $\mathrm{J}$ Dent Hyg. 2009; 7(2):102-107.

14. Nascimento MC, Barbosa LA, Nobre C, Novaes MR, Bittencour S. Evaluation of self-perception in patients with chronic periodontitis - Pilot Study. Int J Dent. 2011; 10(3):154-160.

15. Christensen LB, Hede B, Nielsen E. A cross-sectional study of oral health and oral health-related quality of life among frail elderly persons on admission to a special oral health care programme in Copenhagen City, Denmark. Gerodontology. 2012;29(2):392-400

16. O'Leary TJ, Drake RB, Naylor JE. The plaque control record. J Periodontol. 1972; 43:38-42.
17. Ainamo J, Bay I. Problems and proposals for recording gingivitis and plaque. Int Dent J. 1975; 25:229-235.

18.Lindhe J, Land NP. Tratado de Periodontia Clínica e Implantodontia Oral. $6^{\text {a }}$.ed. Rio de Janeiro: Editora Guanabara Koogan; 2018.

19. Caton JG, Armitage G, Berglundh T, Chapple LLC, Jepsen $\mathrm{S}$, Kornman KS, et al. A new classification scheme for periodontal and peri-implant diseases and conditions Introduction and key changes from the 1999 classification. J Clin Periodontol. 2018;45 (suppl 20):S1-S8.

20. Muhlemann HR. Tooth mobility. The measuring method. Initial and secondary tooth mobility. J Periodontol. 1954; 25:22-29.

21.Slade GD. Derivation and validation of a short-form ora health impact profile. Community Dent Oral Epidemiol. 1997; 25:284-290.

22. Bastos RS. Impacto das condições de saúde bucal em relação à qualidade de vida de adolescentes escolares de 15 a 19 anos, numa dicotomia econômica, no município de Bauru, São Paulo, em 2009. [Tese de Doutorado]. Bauru: Universidade de São Paulo, 2009. 154p.

23. Simangwa LD, Johansson AK, Johansson A, Minja IK Anne N. Åstrøm AN. Oral impacts on daily performances and its socio-demographic and clinical distribution: a crosssectional study of adolescents living in Maasai population areas, Tanzania. Health and Quality of Life Outcomes. 2020; 18(181):1-11.

24. Oliveira EJP, Rocha VFB, Nogueira DA, Pereira AA Qualidade de vida e condições de saúde bucal de hipertensos e diabéticos em um município do Sudeste Brasileiro. Ciênc. Saúde Colet. 2018; 23(3):763-772.

25. Leão RS, Maior JR, Pereira FC, Monteiro GQ, Moraes SL. Impact of Oral Health and Sociodemographic Factors on Quality of Life: A Cross-sectional Study. J Contemp Dent Pract. 2018; 19(4):438-442.

26.Waerhaug J. Effect of Rough Surfaces Upon Gingival Tissue. J Dent Res. 1956; 35(2):323-325.

27. Brasil. Ministério da Saúde. Pesquisa Nacional de Saúde Bucal: resultados principais. Secretaria de Atenção à Saúde. Secretaria de Vigilância em Saúde. Brasília: Ministério da Saúde. 2012.

28. Oliveira GGC, Souza MC, Santos CS, Mattos-Guarald AL, Brito F, Hirata Júnior R. Aspectos antropológicos, bioquímicos e clínicos do cálculo dentário: uma revisão de literatura. Rev Fluminense de Odontologia. 2017; 48:1-12. 
29. Bath-Balogh M, Fehrenbach MJ. Anatomia, histologia e embriologia dos dentes e das estruturas orofaciais. Rio de Janeiro: Elsevier, 2012.

\section{CORRESPONDÊNCIA}

Adriana de Fátima Vasconcelos Pereira

Universidade Federal do Maranhão

Centro de Ciências Biológicas e da Saúde, Departamento de

Odontologia II.

Av. Portugueses, 1966, Campus Dom Delgado,

São Luís, MA - Brasil - CEP 65085-580

Email: adriana.vasconcelos@ufma.br 